\title{
THE BETTI NUMBERS OF THE MODULI SPACE OF STABLE RANK 3 HIGGS BUNDLES ON A RIEMANN SURFACE
}

\author{
PETER B. GOTHEN
}

\section{INTRODUCTION}

In this paper we calculate the Poincaré polynomial of the moduli space of stable Higgs bundles of rank 3 on a Riemann surface $\Sigma$ of genus $g \geq 2$. This space was introduced by Hitchin in [10] and we generalise his calculation in the rank 2 case. We consider Higgs bundles with rank and degree coprime; this condition ensures that the moduli space is smooth. We shall do the calculation for the case of bundles with fixed determinant but we also state the result for bundles with any determinant. From a principal bundle point of view the natural space to consider is the moduli space of $\operatorname{PSL}(3, \mathbb{C})$ Higgs bundles. However, as noted in $[10, \S 5]$, this space has singularities and the method of calculation does not apply directly.

Hitchin analyses many aspects of the geometry of $\mathcal{M}$ in [10], and among other things he shows that $\mathcal{M}$ is a hyperkähler manifold, i.e. it has complex structures $I, J$, and $K$ which satisfy the identities of the quaternions. The complex structure $I$ arises from the interpretation of $\mathcal{M}$ as the moduli space of Higgs bundles. The complex stucture $\mathrm{J}$ arises from an alternative description of $\mathcal{M}$, which follows from results of Donaldson [7] and, more generally, Corlette [5] (see also [10]). There is a universal central extension $\Gamma$ of $\pi_{1} \Sigma$, generated by elements $A_{1}, B_{1}, \ldots, A_{g}, B_{g}$ and a central element $J$ subject to the relation $\prod\left[A_{i}, B_{i}\right]=J$, and $\mathcal{M}$ is the moduli space of irreducible representations of $\Gamma$ in $S L(3, \mathbb{C})$ which take $J$ to a fixed non-trivial central element determined by the first Chern class of the bundle. Thus our calculation gives the Betti numbers of this purely topologically defined space.

As mentioned before our calculation is modeled Hitchin's calculation in the rank 2 case. It exploits the fact that the moduli space has a circle action which respects the symplectic form of the complex structure $I$. There is a moment map for this action, and this is used as a Morse function. Frankel [8] has shown that such a Morse function is perfect,

1991 Mathematics Subject Classification. Primary 14D20, Secondary 14H60,32G13.

Supported by the Danish Research Academy and the Danish Natural Science Research Council. 
thus giving the Poincaré polynomial of the moduli space. Also, it follows from the moment map interpretation that the critical points can be identified with the fixed points of the circle action, and this is what allows them to be described in an explicit way. In [10] the description of the critical submanifolds is quite straightforward, but in our case it becomes more complicated. It is crucial to observe that the critical submanifolds can be described in terms of $\sigma$-stable pairs (see Section 2). These were introduced by Bradlow in [2] and [3], and a careful study in the rank 2 case was made by Thaddeus [14] (see also [4]); these results shall be very useful to us.

The paper is organised as follows: in Section 1 we introduce some terminology and state our main result, which appears as Theorem 1.2. We also outline the structure of the proof. The rest of the paper contains the details of the proof: in Section 2 and Section 3 we describe the critical submanifolds in two different cases, and in Section 4 we calculate the indices of the critical submanifolds.

I would like to thank Nigel Hitchin for introducing me to the subject and for illuminating discussions.

\section{The Moduli SPACE AND Morse TheORY}

First we recall a few facts about Higgs bundles; the reader is referred to [10] for details. Let $\Sigma$ be a closed compact Riemann surface of genus $g$, and denote the canonical bundle by K. A Higgs bundle is a pair $(E, \Phi)$ consisting of a holomorphic vector bundle $E \rightarrow \Sigma$ and a twisted holomorphic endomorphism $\Phi \in H^{0}(\Sigma$; End $(E) \otimes K)$. We denote the rank of $E$ by $k$ and the degree of $E$ by $d$. For any holomorphic bundle $E$ the slope is defined by $\mu(E)=d / k$. A subbundle $F \subset E$ is said to be $\Phi$-invariant if $\Phi(F) \subset F \otimes K$. A Higgs bundle $(E, \Phi)$ is said to be stable if for any proper, non-zero $\Phi$-invariant subbundle $F \subset E$ we have $\mu(F)<\mu(E)$. Let $E \rightarrow \Sigma$ be a $C^{\infty}$ complex vector bundle, and equip $E$ with a Hermitian metric. Then there is a 1-1 correspondence between unitary connections and holomorphic structures on $E$, and for a unitary connection $A$ we denote the $\bar{\partial}$-operator of the corresponding holomorphic structure by $\bar{\partial}_{A}$. We shall consider bundles with a fixed connection $A_{0}$ on the determinant bundle $\Lambda^{k} E$, and denote the corresponding holomorphic determinant bundle by $\Lambda_{0}=\left(\Lambda^{k} E, \bar{\partial}_{A_{0}}\right)$. The space of all such connections is an affine space $\mathcal{A}$ modeled on $\Omega^{1}(\Sigma$; ad $P)$, where ad $P \subset \operatorname{End}(E)$ is the $\mathfrak{s u}(k)$-bundle associated to the adjoint representation of the group $U(k) / Z(U(k))$. Denote the space $\Omega^{1,0}($ ad $P \otimes \mathbb{C})$ of Higgs fields by $\Omega$. For any pair $(A, \Phi) \in \mathcal{A} \times \Omega$, we have the equations

$$
\left\{\begin{array}{l}
F(A)^{\perp}+\left[\Phi, \Phi^{*}\right]=0 \\
\bar{\partial}_{A} \Phi=0
\end{array}\right.
$$


where $F(A)^{\perp}$ denotes the trace free part of the curvature of $A$. The following theorem of Hitchin is of fundamental importance.

Theorem (Hitchin, [10, p.80]). Let $E \rightarrow \Sigma$ be a smooth vector bundle with $(d, k)=1$. There is a bijection between the set of solutions $(A, \Phi)$ with fixed induced connection $A_{0}$ on $\Lambda^{k} E$ to (1.1), modulo $S U(k)$-gauge equivalence, and the set of stable Higgs bundles $\left(\bar{\partial}_{E}, \Phi\right)$ with fixed determinant bundle $\Lambda_{0}$, modulo $S L(k, \mathbb{C})$-gauge equivalence.

When $(d, k)=1$ Hitchin also constructs a moduli space, $\mathcal{M}$, of stable Higgs bundles with fixed determinant. This is done by gauge theoretic methods, and the construction shows that $\mathcal{M}$ is a smooth manifold of dimension $4(g-1)\left(k^{2}-1\right)([10, p .87])$. The results of [10] are only stated in the case of bundles of rank 2 , but the generalisation to arbitrary rank poses no significant problems. Alternatively one could refer to Simpson's theorem [13]: he proves analogous results for vector bundles on Kähler manifolds of arbitrary dimension, but we do not really need these more general results.

We can now state our main result.

Theorem 1.2. Let $\Sigma$ be a closed Riemann surface of genus $g \geq 2$, and let $\Lambda_{0}$ be a holomorphic line bundle on $\Sigma$ of degree $d$ with $(d, 3)=1$. Let $\mathcal{M}$ be the moduli space of rank 3 stable Higgs bundles on $\Sigma$ with fixed determinant bundle $\Lambda_{0}$. The Poincaré polynomial of $\mathcal{M}$ is

$$
\begin{gathered}
P_{t}(\mathcal{M})=\frac{(1+t)^{4 g-4}}{(1-t)^{4}}\left(2 t^{2}+t^{4}+2 t^{2 g}+2 t^{2 g+2}-\frac{1}{4} t^{4 g-4}-3 g t^{4 g-3}\right. \\
\left.+\left(6 g^{2}+2 g-3\right) t^{4 g-2}+\left(11 g-12 g^{2}\right) t^{4 g-1}+\left(6 g^{2}-10 g+\frac{17}{4}\right) t^{4 g}-t^{8 g-6}-t^{10 g-8}\right) \\
+\frac{t^{2 g}(1+t)^{2 g-4}}{(1-t)^{4}\left(1+t^{2}\right)^{2}}\left((1+t)^{2 g}\left(-2 t^{4}-2 t^{6}+t^{2 g-4}+2 t^{2 g-2}+t^{2 g}-t^{4 g-2}\right)\right. \\
\left.+t^{6 g-8}\left(1+t^{3}\right)^{2 g}\left(-2 g-t^{2}+(2 g-2) t^{4}\right)\right) \\
-\frac{2^{2 g} t^{2 g}(1+t)^{2 g-1}}{(1-t)^{4}}+\frac{2 g t^{8 g-8}(1+t)^{2 g-3}\left(1+t^{3}\right)^{2 g-1}}{(1-t)^{3}\left(1+t^{2}\right)}+\frac{2^{2 g-1} t^{10 g-8}(1+t)^{2 g}}{(1-t)^{3}\left(1-t^{3}\right)} \\
+\frac{t^{4 g-4}(1-t)^{2 g-1}(1+t)^{2 g-1}}{4\left(1+t^{2}\right)}+\frac{t^{6 g-2}(1+t)^{4 g-3}\left(1+t^{2}+t^{4}\right)}{(t-1)^{3}\left(1+t^{2}\right)^{2}\left(t^{6}-1\right)} \\
+\frac{\left(1+t^{5}\right)^{2 g}\left(1+t^{3}\right)^{2 g-1}}{\left(t^{2}-1\right)\left(t^{4}-1\right)^{2}\left(t^{3}-1\right)}+t^{4 g-4}\left(\left(3^{2 g}-1\right)(1+t)^{4 g-4}-3^{2 g}\right) .
\end{gathered}
$$

Let $\mathcal{M}^{\prime}$ denote the moduli space of stable Higgs bundles of rank 3 and degree $d$ with $(d, 3)=1$ and any determinant. Then

$$
P_{t}\left(\mathcal{M}^{\prime}\right)=(1+t)^{2 g}\left(P_{t}(\mathcal{M})-\left(3^{2 g}-1\right) t^{4 g-4}\left((1+t)^{4 g-4}-1\right)\right) .
$$

It is interesting to note that the Poincaré polynomial of $\mathcal{M}^{\prime}$ does not split as the product of those of the Jacobian and $\mathcal{M}$. This is in 
contrast to the case of stable bundles (without Higgs field), see [1]. In particular it follows that tensoring by a linebundle gives a nontrivial action of the group

$$
\Gamma_{3}=\left\{L \in \operatorname{Jac}^{0}(\Sigma) \mid L^{3}=\mathcal{O}\right\} \cong(\mathbb{Z} / 3)^{2 g}
$$

on $H^{*}(\mathcal{M} ; \mathbb{Q})$.

Some simpler results can be obtained from the above formulas. Setting $t=-1$ we see that $\chi(\mathcal{M})=-3^{2 g}$, while $\chi\left(\mathcal{M}^{\prime}\right)=0$. And for a Riemann surface of genus 2 , the above formula becomes:

$$
\begin{aligned}
P_{t}(\mathcal{M}) & =1+3 t^{2}+20 t^{3}+54 t^{4}+416 t^{5}+572 t^{6}+376 t^{7}+117 t^{8} \\
& +32 t^{9}+47 t^{10}+56 t^{11}+42 t^{12}+28 t^{13}+16 t^{14}+8 t^{15}+3 t^{16} .
\end{aligned}
$$

The proof of the theorem follows the method of $[10, \S 7]$. It uses some of the extra structure $\mathcal{M}$ gets from the gauge theory construction. From the identification $\Omega^{1}(\Sigma$; ad $P)=\Omega^{0,1}(\Sigma$; ad $P \otimes \mathbb{C}$ ) (given by the correspondence between holomorphic structures and unitary connections) we see that $\mathcal{A} \times \Omega$ is a complex vector space. This gives an almost complex structure $I$ on $\mathcal{M}$, and this turns out to be integrable (as mentioned above, $\mathcal{M}$ is in fact a hyperkähler manifold). A Kähler metric on $\mathcal{A} \times \Omega$ is defined by

$$
g\left(\left(\psi_{1}, \Phi_{1}\right),\left(\psi_{2}, \Phi_{2}\right)\right)=2 i \int_{\Sigma} \operatorname{tr}\left(\psi_{1}^{*} \psi_{2}+\Phi_{1} \Phi_{2}^{*}\right),
$$

and because it is invariant under the action of the gauge group, it descends to $\mathcal{M}$. The corresponding Kähler form is $\omega_{1}(X, Y)=g(I X, Y)$. Furthermore $S^{1}$ acts on $\mathcal{M}$ by $(A, \Phi) \mapsto\left(A, e^{i \theta} \Phi\right)$, preserving $g$ and the symplectic form $\omega_{1}$. The function $(A, \Phi) \mapsto-\frac{1}{2}\|\Phi\|^{2}$ is a moment map for the $S^{1}$-action with respect to the symplectic form $\omega_{1}$. This is basically the Morse function we shall use, but we choose to normalise it as

$$
\mu(A, \Phi)=\frac{1}{4 \pi}\|\Phi\|^{2}
$$

Frankel [8, p.5] shows that in this situation the function $\mu$ is a nondegenerate perfect Morse function. Therefore the Poincaré polynomial of the moduli space $\mathcal{M}$ is given by the Morse counting polynomial

$$
P_{t}(\mathcal{M})=\sum_{N} t^{\lambda_{N}} P_{t}(N)
$$

where the sum is over the critical submanifolds, and $\lambda_{N}$ is the index of the critical submanifold $N$, i.e. the dimension of the subbundle of the normal bundle, on which the Hessian of $\mu$ is negative definite. So we must determine the critical submanifolds and calculate their Poincaré polynomials and indices.

It follows from the moment map interpretation of $\mu$ that the critical points of $\mu$ are exactly the fixed points of the $S^{1}$-action on $\mathcal{M}$. Let $(A, \Phi) \in \mathcal{A} \times \Omega$ represent a fixed point of the $S^{1}$-action. If $(A, \Phi)=$ 
( $\left.A, e^{i \theta} \Phi\right)$ we must have $\Phi=0$. The corresponding critical submanifold is $N_{0}=\mu^{-1}(0)$, the moduli space of stable bundles of rank $k$. The index of $N_{0}$ is of course $\lambda_{N_{0}}=0$, and Desale and Ramanan calculated the Poincaré polynomial of $N_{0}$ in [6, p.241]. Their formula is

$$
\begin{aligned}
& \quad P_{t}\left(N_{0}\right)= \\
& \frac{\left(t^{5}+1\right)^{2 g}\left(t^{3}+1\right)^{2 g}-\left(t^{2}+1\right)^{2} t^{4 g-2}(1+t)^{2 g}\left(1+t^{3}\right)^{2 g}+\left(1+t^{2}+t^{4}\right) t^{6 g-2}(1+t)^{4 g}}{\left(t^{2}-1\right)\left(t^{4}-1\right)^{2}\left(t^{6}-1\right)} .
\end{aligned}
$$

The other critical submanifolds are described in propositions 2.5, 2.9, and 3.10, while their indices are given in Proposition 4.2. From these results an elementary but rather unpleasant calculation gives the formula of Theorem 1.2, and we shall omit the details.

To determine the other critical submanifolds, observe that if $(A, \Phi)$ represents a fixed point and $\Phi \neq 0$ then there is a gauge transformation taking $(A, \Phi)$ to $\left(A, e^{i \theta} \Phi\right)$. As noted in [11, p.466] this gives an infinitesimal gauge transformation $\psi$, which splits the bundle $E$ into eigenspaces $E=\bigoplus_{m} U_{m}$, where $\psi$ acts by $i m$ on $U_{m}$, for real numbers $m$. With respect to this decomposition

$$
\Phi: U_{m} \rightarrow U_{m+1} \otimes K,
$$

and all these maps are non-trivial. Furthermore the consecutive values of $m$ differ by 1 . Thus the critical submanifolds fall in different families according to how the bundle $E$ splits into eigenspaces. From now on we shall assume that $k=3$, and in this case there are three distinct types of critical points: we shall say that a Higgs bundle $(E, \Phi)$ (or the critical point it represents) is of type $(1,2)$ if it is of the form $E=L \oplus V$, where $\operatorname{rk}(L)=1$ and $\operatorname{rk}(V)=2$, and where

$$
\Phi=\left(\begin{array}{ll}
0 & 0 \\
\phi & 0
\end{array}\right)
$$

with $\phi: L \rightarrow V \otimes K$. Similarly we say that $(E, \Phi)$ is of type $(2,1)$ if it is of the form $E=V \oplus L$, with $\operatorname{rk}(V)=2$ and $\operatorname{rk}(L)=1$, and where

$$
\Phi=\left(\begin{array}{ll}
0 & 0 \\
\phi & 0
\end{array}\right)
$$

with $\phi: V \rightarrow L K$. Finally, $(E, \Phi)$ is said to be of type $(1,1,1)$, if it is of the form $E=L_{1} \oplus L_{2} \oplus L_{3}$, with

$$
\Phi=\left(\begin{array}{ccc}
0 & 0 & 0 \\
\phi_{1} & 0 & 0 \\
0 & \phi_{2} & 0
\end{array}\right)
$$

where $\phi_{1}: L_{1} \rightarrow L_{2} K$ and $\phi_{2}: L_{2} \rightarrow L_{3} K$.

In the following two sections we shall give an explicit description of the different types of critical submanifolds. 


\section{Critical submanifolds of type $(1,2)$ And $(2,1)$}

Let $(E, \Phi)$ represent a critical point of $\mu$ of type $(1,2)$, and write $E=$ $L \oplus V$, and $\Phi=\left(\begin{array}{ll}0 & 0 \\ \phi & 0\end{array}\right)$. Set $d=\operatorname{deg}(E), l=\operatorname{deg}(L)$, and $v=\operatorname{deg}(V)$, then $d=l+v$. An easy calculation using the Higgs bundle equation $F(A)^{\perp}+\left[\Phi, \Phi^{*}\right]=0$ and the fact that $F(A)=F(A)^{\perp}+\frac{1}{3} F\left(A_{0}\right) \cdot I$, shows that the (critical) value of $\mu$ at the point represented by $(E, \Phi)$ is

$$
\mu=l-\frac{1}{3} d
$$

The fact that $(E, \Phi)$ is a stable Higgs bundle allows us to get bounds on the values $l$ (and hence $\mu$ ) can attain. There is a rank 1 subbundle $L^{\prime} \subset V$, defined by the requirement that $\phi: L \rightarrow L^{\prime} K$. Thus $\phi$ is a section of the line bundle $L^{-1} L^{\prime} K$. There are three obvious $\Phi$-invariant subbundles of $E$, namely $L^{\prime}, L \oplus L^{\prime}$, and $V$. Applying the stability condition to these and combining with the inequality $\operatorname{deg}\left(L^{-1} L^{\prime} K\right)>0$ ( $\phi$ is a non-zero section of $L^{-1} L^{\prime} K$ ) gives

$$
\frac{1}{3} d<l<\frac{1}{3} d+g-1 .
$$

It follows that we can construct any Higgs bundle representing a critical point of type $(1,2)$ by first choosing a holomorphic line bundle $L$ of degree $l$ with $\frac{1}{3} d<l<\frac{1}{3} d+g-1$. Then we choose a rank 2 bundle $V$ and a non-zero section $\phi \in H^{0}\left(\Sigma ; L^{-1} V K\right)$ such that $\Lambda_{0}=\Lambda^{3}(L \oplus$ $V)$, and set $E=L \oplus V$ and $\Phi=\left(\begin{array}{ll}0 & 0 \\ \phi & 0\end{array}\right)$. But not any $V$ and $\phi$ will do; they have to be chosen such that $(E, \Phi)$ becomes a stable Higgs bundle. Conversely the calculation of [11, p.464] shows that any stable Higgs bundle constructed in this way represents a critical point of $\mu$. As we shall show later, the condition on $V$ and $\phi$ turns out to be essentially Bradlow's condition of $\tau$-stability (see [2] and [3]). In the case of bundles of rank 2 on a Riemann surface it takes the following form (cf. [14, p.3]).

Definition 2.3. Let $\sigma$ be a positive rational number. A pair $(\tilde{V}, \phi)$ consisting of a holomorphic bundle $\tilde{V} \rightarrow \Sigma$ and a non-zero section $\phi \in H^{0}(\Sigma ; \tilde{V})$ is said to be $\sigma$-semi-stable if for any line bundle $\tilde{U} \subset \tilde{V}$

$$
\operatorname{deg}(\tilde{U}) \leq \frac{1}{2} \operatorname{deg}(\tilde{V})-\sigma \quad \text { if } \phi \in H^{0}(\Sigma ; \tilde{U})
$$

and

$$
\operatorname{deg}(\tilde{U}) \leq \frac{1}{2} \operatorname{deg}(\tilde{V})+\sigma \quad \text { if } \phi \notin H^{0}(\Sigma ; \tilde{U}) .
$$

If we have strict inequality above $(\tilde{V}, \phi)$ is said to be $\sigma$-stable.

Let $r=\operatorname{deg}(\tilde{V})$. For $\sigma \not \equiv \frac{1}{2} r(\bmod \mathbb{Z})$ and $\sigma<\frac{1}{2} r$ smooth moduli spaces of $\sigma$-stable pairs can be constructed; in [14] Thaddeus constructed a moduli space $\mathcal{N}(\sigma, \Lambda)$ of pairs with fixed determinant bundle $\Lambda$ by geometric invariant theory, and in [3] Bradlow and Daskalopoulos constructed a moduli space $\mathcal{N}(\sigma)$ of pairs with any determinant bundle (of degree $r$ ). 
Before we can apply this to our situation, we need a lemma about $\Phi$-invariant subbundles.

Lemma 2.4. Let $(E, \Phi)$ be a Higgs bundle constructed from $L, V$, and $\phi$ as described above. If $U \subset E$ is a $\Phi$-invariant subbundle, not contained in $V$, then $L^{\prime} \subset U$.

Proof. Because $U$ is not contained in $V$, the projection $\pi: U \rightarrow L$ is generically non-zero. Hence $\Phi_{\mid U}: U \rightarrow L^{\prime} K$ is generically non-zero. By the $\Phi$-invariance of $U$ it follows that $L^{\prime} K$ is contained in $U K$ generically, and hence, by continuity, identically. This proves the lemma.

We can now determine the critical submanifolds.

Proposition 2.5. Let $r=d-3 l+4 g-4$ and let $h: \operatorname{Jac}^{l}(\Sigma) \rightarrow \operatorname{Jac}^{r}(\Sigma)$ be the $3^{2 g}$-fold covering of the Jacobian given by $L \mapsto L^{-3} \Lambda_{0} K^{2}$. Set $\sigma=-\frac{1}{6} d+\frac{1}{2} l$. For $\frac{1}{3} d<l<\frac{1}{3} d+g-1$ the critical submanifold $N\left(l-\frac{1}{3} d\right)$ corresponding to the critical value $\mu=l-\frac{1}{3} d$ is the pull-back of this covering under the map $\mathcal{N}(\sigma) \stackrel{\text { det }}{\longrightarrow} \operatorname{Jac}(\Sigma)$. Consequently there is a pull-back diagram

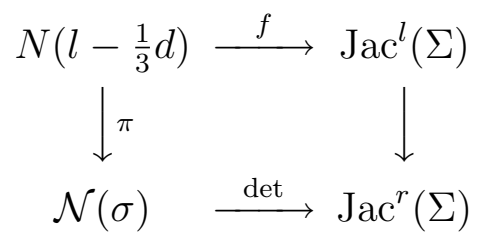

The map $f$ is given by $(L \oplus V, \Phi) \mapsto L$, and the map $\pi$ is given by $(L \oplus V, \Phi) \mapsto\left(L^{-1} V K, \phi\right)$, where $\Phi=\left(\begin{array}{ll}0 & 0 \\ \phi & 0\end{array}\right)$.

Proof. Let $L$ be any line bundle of degree $l$. Consider pairs $(V, \phi)$, where $V$ is a rank 2 bundle with $\Lambda^{2} V=L^{-1} \Lambda_{0}$ and $\phi \in H^{0}\left(\Sigma ; L^{-1} V K\right)-0$. We need to describe all such pairs, such that the Higgs bundle, defined by setting $E=L \oplus V$ and $\Phi=\left(\begin{array}{ll}0 & 0 \\ \phi & 0\end{array}\right)$, is stable. Let $L^{\prime} \subset V$ be the line bundle defined by the requirement that $\phi: L \rightarrow L^{\prime} K$.

First we consider $\Phi$-invariant line bundles $U \subset E$. Note that $U \subset V$, because otherwise $U=L^{\prime}$ by Lemma 2.4, in contradiction with $U \not \subset V$. If $U=L^{\prime}$ the bundle $L \oplus L^{\prime}$ is $\Phi$-invariant, and hence we get the condition

$$
\operatorname{deg}(U)<\frac{2}{3} d-l
$$

for stability of $(E, \Phi)$. On the other hand, if $U \neq L$, we simply get the condition

$$
\operatorname{deg}(U)<\frac{1}{3} d .
$$

To apply Bradlow's $\sigma$-stability condition we note that we can equivalently consider pairs $(\tilde{V}, \phi)$ with $\tilde{V}=L^{-1} V K$ and $\phi \in H^{0}(\Sigma ; \tilde{V})-0$, and such that $\tilde{V}$ has fixed determinant bundle $\Lambda^{2} \tilde{V}=\Lambda_{0} L^{-3} K^{2}$. For 
a line bundle $U \subset V$, let $\tilde{U}=L^{-1} U K$ be the corresponding subbundle of $\tilde{V}$. The conditions (2.6) and (2.7) are equivalent to

$$
\operatorname{deg}(\tilde{U})<\frac{2}{3} d-2 l+2 g-2 \text { if } \phi \in H^{0}(\Sigma ; \tilde{U})
$$

and

$$
\operatorname{deg}(\tilde{U})<\frac{1}{3} d-l+2 g-2 \text { if } \phi \notin H^{0}(\Sigma ; \tilde{U}) .
$$

Setting $\sigma=-\frac{1}{6} d+\frac{1}{2} l$, this is just the condition that $(\tilde{V}, \Phi)$ be $\sigma$-stable.

Actually this is sufficient to ensure stability of $(E, \Phi)$ : consider any $\Phi$-invariant bundle $U \subset E$ of rank 2 . If $U=V$ the stability condition is satisfied by construction, so we may assume that the restriction of the projection $\pi_{L}: L \oplus V \rightarrow L$ to $U$ is non-zero. By Lemma $2.4 L^{\prime} \subset U$, and as $L^{\prime} \subset \operatorname{ker}\left(\pi_{L}\right)$ we get a non-zero map $U / L^{\prime} \rightarrow L$. Hence $\operatorname{deg}(U) \leq$ $\operatorname{deg}(L)+\operatorname{deg}\left(L^{\prime}\right)$, and we are done by (2.6), which is equivalent to $\operatorname{deg}(L)+\operatorname{deg}\left(L^{\prime}\right)<\frac{2}{3} d$.

From this description it is clear that $N\left(l-\frac{1}{3} d\right)$ is the pull-back by $\mathcal{N}(\sigma) \stackrel{\text { det }}{\longrightarrow} \operatorname{Jac}^{r}(\Sigma)$ of the $3^{2 g}$-fold covering $\operatorname{Jac}^{l}(\Sigma) \rightarrow \operatorname{Jac}^{r}(\Sigma)$ given by $L \mapsto L^{-3} \Lambda_{0} K^{2}$. This finishes the proof.

Finally we need to calculate the Poincaré polynomial of the critical submanifold $N\left(l-\frac{1}{3} d\right)$. This is done along the lines of $[14,(4.1)]$; we briefly recall the relevant details (or rather the version for $\sigma$-stable pairs without fixed determinant of [4]). Let $i$ be an integer in the interval $[0,(r-1) / 2]$, then the $\sigma$-stability condition is the same for all $\sigma \in(\max (0, r / 2-i-1), r / 2-i)$. Put $\mathcal{N}_{i}=\mathcal{N}(\sigma)$ for $\sigma$ in this interval. There are subvarieties $\mathbb{P} W_{i}^{+}$of $\mathcal{N}_{i}$ and $\mathbb{P} W_{i}^{-}$of $\mathcal{N}_{i-1}$, such that when these are blown up, we get the same variety $\tilde{\mathcal{N}}_{i}$. Furthermore $\mathbb{P} W_{i}^{+}$is a $\mathbb{P}^{r+g-2-2 i}$-bundle over $S^{i} \Sigma \times \operatorname{Jac}^{r-i}(\Sigma)$ and $\mathbb{P} W_{i}^{-}$is a $\mathbb{P}^{i-1}$-bundle over $S^{i} \Sigma \times \operatorname{Jac}^{r-i}(\Sigma)$. Also, if the projection $\pi: \mathbb{P} W_{i}^{+} \rightarrow S^{i} \Sigma \times \operatorname{Jac}^{r-i}(\Sigma)$ is composed with the map $S^{i} \Sigma \times \operatorname{Jac}^{r-i}(\Sigma):(D, L) \mapsto[D] \otimes L$ we get the determinant map (and similarly for $\mathbb{P} W_{i}^{-}$).

Proposition 2.8. The Poincaré polynomial of $N\left(l-\frac{1}{3} d\right)$ is

$P_{t}\left(N\left(l-\frac{1}{3} d\right)\right)=\frac{(1+t)^{2 g}}{1-t^{2}} \operatorname{Coeff}_{x^{i}}\left(\frac{t^{2 d-6 l+10 g-10-4 i}}{x t^{4}-1}-\frac{t^{2 i+2}}{x-t^{2}}\right)\left(\frac{(1+x t)^{2 g}}{(1-x)\left(1-x t^{2}\right)}\right)$,

where $i=\left[\frac{2}{3} d\right]-2 l+2 g-2$.

Proof. Note that $i=\left[\frac{2}{3} d\right]-2 l+2 g-2$ corresponds to $\sigma=-\frac{1}{6} d+\frac{1}{2} l$. Let $N_{i}$ be defined by the following pull-back diagram

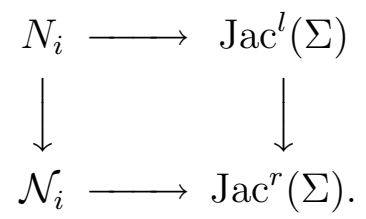

We can similarly pull back the subvariety $\mathbb{P} W_{i}^{+}$of $\mathcal{N}_{i}$ to get a subvariety $\left(\mathbb{P} W_{i}^{+}\right)^{\prime}$ of $N_{i}$. Blowing up this we get a new variety $\tilde{N}_{i}$. But this is 
the same variety as the one obtained by pulling back the blow-up $\tilde{\mathcal{N}}_{i}$, because they are locally isomorphic, and blowing up doesn't change the fundamental group. Note also that $\left(\mathbb{P} W_{i}^{+}\right)^{\prime}$ is a $\mathbb{P}^{r+g-2-2 i}$-bundle over the pull-back of $\operatorname{Jac}^{l}(\Sigma) \rightarrow \operatorname{Jac}^{r}(\Sigma)$ under the map $S^{i} \Sigma \times \operatorname{Jac}^{r-i}(\Sigma) \rightarrow$ $\operatorname{Jac}^{r}(\Sigma):(D, L) \mapsto[D] \otimes L$. It is, however, easy to see that this pullback is isomorphic to $S^{i} \Sigma \times \mathrm{Jac}^{r-i}(\Sigma)$. Of course similar remarks apply to $\mathbb{P} W_{i}^{-}$. Finally we make the observation that $N_{0}$ is a $\mathbb{P}^{r+g-2}$-bundle over $\operatorname{Jac}^{r}(\Sigma)$. Altogether this information allows us to replicate the argument of $[14,(4.1)]$ and arrive at the formula stated. It should be remarked that the basic reason why the calculation works and no further informaton about the various projective bundles is needed, is that the Poincaré polynomial of any projective bundle splits.

The description of critical submanifolds of type $(2,1)$ is of course completely analogous. Alternatively one can note that $E \mapsto E^{*}$ takes stable Higgs bundles of type $(1,2)$ to stable Higgs bundles of type $(2,1)$. In any case we have the following.

Proposition 2.9. For $\frac{1}{3} d+1-g<\bar{l}<\frac{1}{3} d$ there are critical submanifolds $N\left(\frac{1}{3} d-\bar{l}\right)$ corresponding to the critical value $\mu=\frac{1}{3} d-\bar{l}$. These fit into a pull-back diagram

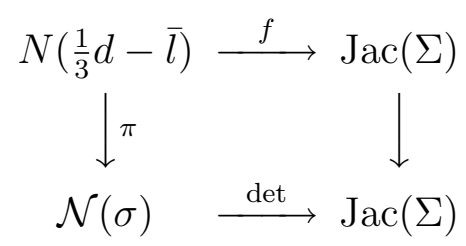

where $\sigma=\frac{1}{6} d-\frac{1}{2} \bar{l}$. The map $f$ is given by $(V \oplus L, \Phi) \mapsto L$, and the map $\pi$ is given by $(V \oplus L, \Phi) \mapsto\left(V^{*} L K, \phi\right)$, where $\Phi=\left(\begin{array}{ll}0 & 0 \\ \phi & 0\end{array}\right)$. The map on the Jacobians is given by $L \mapsto \Lambda_{0}^{-1} L^{3} K^{2}$.

Furthermore the Poincaré polynomial of $N\left(\frac{1}{3} d-\bar{l}\right)$ is given by

$P_{t}\left(N\left(\frac{1}{3} d-\bar{l}\right)\right)=\frac{(1+t)^{2 g}}{1-t^{2}} \operatorname{Coeff}_{x^{i}}\left(\frac{t^{6 \bar{l}-2 d+10 g-10-4 i}}{x t^{4}-1}-\frac{t^{2 i+2}}{x-t^{2}}\right)\left(\frac{(1+x t)^{2 g}}{(1-x)\left(1-x t^{2}\right)}\right)$,

where $i=\left[-\frac{2}{3} d\right]+2 \bar{l}+2 g-2$.

\section{Critical submanifolds of type $(1,1,1)$}

The remaining case is when $(E, \Phi)$ is a critical point of type $(1,1,1)$, i.e. it is of the form

$$
E=L_{1} \oplus L_{2} \oplus L_{3}
$$

with

$$
\Phi=\left(\begin{array}{ccc}
0 & 0 & 0 \\
\phi_{1} & 0 & 0 \\
0 & \phi_{2} & 0
\end{array}\right)
$$


where $\phi_{1}: L_{1} \rightarrow L_{2} K$ and $\phi_{2}: L_{2} \rightarrow L_{3} K$ are non-zero. It will be convenient to introduce

$$
\begin{aligned}
& M_{1}=L_{1}^{-1} L_{2} K, \\
& M_{2}=L_{2}^{-1} L_{3} K,
\end{aligned}
$$

so that $\phi_{1} \in H^{0}\left(\Sigma ; M_{1}\right)$ and $\phi_{2} \in H^{0}\left(\Sigma ; M_{2}\right)$. Set $l_{i}=\operatorname{deg}\left(L_{i}\right), m_{i}=$ $\operatorname{deg}\left(M_{i}\right)$, and $m=m_{1}+m_{2}$. The value of $\mu$ at a critical point can be calculated to be

$$
\mu=4(g-1)-m \text {. }
$$

Now, clearly $d=l_{1}+l_{2}+l_{3}$ and because $M_{1}$ and $M_{2}$ have non-zero global sections we have

$$
m_{i} \geq 0, \quad \text { for } i=1,2
$$

There are two subbundles which are clearly $\Phi$-invariant, namely $L_{2} \oplus L_{3}$ and $L_{3}$. Hence by stability we get

$$
\begin{aligned}
l_{3} & <\frac{1}{3} d, \\
l_{2}+l_{3} & <\frac{2}{3} d .
\end{aligned}
$$

The $l_{i}$ 's can be expressed in terms of the $m_{i}$ 's (and vice versa), and the inequalities (3.3) and (3.4) are equivalent to

$$
\begin{aligned}
& m_{1}+2 m_{2}<6(g-1), \\
& 2 m_{1}+m_{2}<6(g-1) .
\end{aligned}
$$

Note also that the values of $m$ lie in the range $0 \leq m<4(g-1)$. Hence, for $m$ in this range, there are critical submanifolds $N(4(g-1)-m)$ corresponding to the critical value $\mu=4(g-1)-m$. These have components $N\left(m_{1}, m_{2}\right)$ for $m=m_{1}+m_{2}$, and $m_{1}$ and $m_{2}$ positive and satisfying (3.5) and (3.6).

The line bundles $M_{1}$ and $M_{2}$ do not quite determine $L_{1}, L_{2}$, and $L_{3}$. But a small calculation shows that

$$
\begin{aligned}
& L_{1}=M_{1}^{-1} L_{2} K, \\
& L_{3}=M_{2} L_{2} K^{-1}, \\
& L_{2}^{3}=M_{1} M_{2}^{-1} \Lambda_{0} .
\end{aligned}
$$

We see from the above that any critical point in $N\left(m_{1}, m_{2}\right)$ can be constructed in the following way: first we choose line bundles $M_{1}$ and $M_{2}$ of degrees $m_{1}$ and $m_{2}$ respectively, and non-zero sections $\phi_{i} \in$ $H^{0}\left(\Sigma ; M_{i}\right)$ for $i=1,2$. This can be done by choosing effective divisors $D_{i}$ of degrees $m_{i}$ on $\Sigma$, i.e. points of the symmetric products $S^{m_{i}} \Sigma$. The sections $\phi_{i}$ will be determined up to a non-zero constant, so different choices of $\phi_{i}$ will give equivalent solutions. Then we choose one of the $3^{2 g} L_{2}$ 's satisfying (3.9), and define $L_{1}$ and $L_{3}$ by the formulas (3.7) and (3.8). Finally we define $(E, \Phi)$ in the obvious way (by (3.1) and 
(3.2)). Next we shall prove that any Higgs bundle $(E, \Phi)$ constructed in this way is stable.

Proposition 3.10. For $0 \leq m<4(g-1)$ there are critical submanifolds

$$
N(4(g-1)-m)=\coprod_{m_{1}, m_{2}} N\left(m_{1}, m_{2}\right)
$$

corresponding to the critical value $\mu=4(g-1)-m$. These have components $N\left(m_{1}, m_{2}\right)$, for $m_{1}+m_{2}=m, m_{i} \geq 0, m_{1}+2 m_{2}<6(g-1)$, and $2 m_{1}+m_{2}<6(g-1)$. Each $N\left(m_{1}, m_{2}\right)$ is the $3^{2 g}$-fold covering of $S^{m_{1}} \Sigma \times S^{m_{2}} \Sigma$ given by the pull-back of the covering $\operatorname{Jac}(\Sigma) \stackrel{3 \cdot}{\rightarrow} \operatorname{Jac}(\Sigma)$ under the map

$$
\begin{aligned}
f: S^{m_{1}} \Sigma \times S^{m_{2}} \Sigma & \rightarrow \operatorname{Jac}(\Sigma) \\
\left(D_{1}, D_{2}\right) & \mapsto M_{1} M_{2}^{-1} \Lambda_{0} .
\end{aligned}
$$

Here $M_{i}=\left[D_{i}\right]$ is the line bundle associated to the divisor $D_{i}$.

Proof. We must show that $(E, \Phi)$ constructed as above is stable. Denote the projections $E \rightarrow L_{i}$ by $\pi_{i}$ for $i=1,2,3$. Let $U \subset E$ be any $\Phi$-invariant rank 1 subbundle of $E$. Then $\pi_{1 \mid U}$ and $\pi_{2 \mid U}$ cannot both be zero, because then $U=L_{1}$, which is obviously not $\Phi$-invariant. Similarly $\pi_{2 \mid U} \neq 0$ implies $\pi_{3 \mid U} \neq 0$. Hence $\pi: U \rightarrow L_{3}$ is always non-zero, and so $\operatorname{deg}(U)<l_{3}<\frac{1}{3} d$ by $(3.3)$.

Now let $U \subset E$ be a $\Phi$-invariant rank 2 subbundle. If $U=L_{2} \oplus L_{3}$, we are done by (3.4). In fact this is the only $\Phi$-invariant rank 2 subbundle of $E$. To see this assume that $\pi_{1 \mid U} \neq 0$. Then $\left(\pi_{2}+\pi_{3}\right)_{\mid U}: U \rightarrow$ $L_{2} \oplus L_{3}$ is generically, and hence identically of rank 1 . It follows that $U=L_{1} \oplus U^{\prime}$, where $U^{\prime}=\left(\pi_{2}+\pi_{3}\right)(U) \subset L_{2} \oplus L_{3}$. By $\Phi$-invariance of $U$ we get

$$
\Phi\left(L_{1}\right) \subset U^{\prime} K \cap L_{2} K
$$

As $\Phi\left(L_{1}\right) \neq 0$, it follows that $U^{\prime}=L_{2}$, and hence $U=L_{1} \oplus L_{2}$. But this is clearly not $\Phi$-invariant, in contradiction with our assumption.

Finally we need to calculate the Betti numbers of $N\left(m_{1}, m_{2}\right)$.

Proposition 3.11. The Poincaré polynomial of the component $N\left(m_{1}, m_{2}\right)$ of the critical submanifold $N(4(g-1)-m)$ is

$P_{t}\left(N\left(m_{1}, m_{2}\right)\right)=P_{t}\left(S^{m_{1}} \Sigma\right) P_{t}\left(S^{m_{2}} \Sigma\right)+\left(\begin{array}{c}2 g-2 \\ m_{1}\end{array}\right)\left(\begin{array}{c}2 g-2 \\ m_{2}\end{array}\right)\left(3^{2 g}-1\right) t^{m}$.

Proof. $H^{*}\left(N\left(m_{1}, m_{2}\right) ; \mathbb{R}\right)$ is the $S_{m_{1}} \times S_{m_{2}}$-invariant part of $H^{*}\left(\tilde{\Sigma}^{m} ; \mathbb{R}\right)$, where $\tilde{\Sigma}^{m}$ is the covering

$$
(\mathbb{Z} / 3)^{2 g} \rightarrow \tilde{\Sigma}^{m} \rightarrow \Sigma^{m}
$$

of $\Sigma^{m}$ induced by the composite $\Sigma^{m} \rightarrow S^{m_{1}} \Sigma \times S^{m_{2}} \Sigma \stackrel{f}{\rightarrow} \mathrm{Jac}(\Sigma)$. The fundamental group of $\Sigma^{m}$ is $\pi_{1} \Sigma^{m}=\pi_{1} \Sigma \oplus \cdots \oplus \pi_{1} \Sigma$, the direct sum 
of $m$ copies of $\pi_{1} \Sigma$, and we denote the $2 g$ generators of the $r$ th copy by $\left\{\alpha_{i}^{r}\right\}_{i=1}^{2 g}$. In the case $m=1$ the map $f: \Sigma \rightarrow \operatorname{Jac}(\Sigma)$ induces an isomorphism

$$
f^{*}: H^{1}(\operatorname{Jac}(\Sigma) ; \mathbb{Z}) \stackrel{\cong}{\rightrightarrows} H^{1}(\Sigma ; \mathbb{Z}),
$$

and from this it follows that $\alpha_{i}^{r} \in \pi_{1} \Sigma^{m}$ acts on the covering $(\mathbb{Z} / 3)^{2 g} \rightarrow$ $\tilde{\Sigma}^{m} \rightarrow \Sigma^{m}$ by permuting the elements of the $i$ th copy of $\mathbb{Z} / 3$ cyclically. Consequently $H^{*}\left(\tilde{\Sigma}^{m} ; \mathbb{R}\right)=H^{*}\left(\Sigma^{m} ; \mathcal{F}\right)$, where $\mathcal{F}$ is a local coefficient defined as follows. Let $W_{i}^{\prime}$ be the $\pi_{1} \Sigma^{m}$-representation in $\mathbb{R}^{3}$ defined by letting $\alpha_{i}^{r}$ permute the basis vectors of $\mathbb{R}^{3}$, and letting the other $\alpha_{i}^{r}$ act trivially. Then the local coefficient system $\mathcal{F}$ is given by the representation $W_{1}^{\prime} \otimes \cdots \otimes W_{2 g}^{\prime}$. Note that $W_{i}^{\prime}$ splits as $W_{i}^{\prime}=\mathbb{R} \oplus W_{i}$, where $\alpha_{i}^{r}$ acts trivially on $\mathbb{R}$ and rotates the 2 -dimensional space $W_{i}$ through an angle of $\frac{2 \pi}{3}$. Thus $\mathcal{F}$ is of the form

$$
\mathcal{F}=\bigoplus_{j=0}^{2 g} \mathcal{F}_{j},
$$

where $\mathcal{F}_{0}$ is given by the trivial 1 -dimensional real representation and $\mathcal{F}_{j}$ is given by the representation

$$
\bigoplus_{i_{1}<\cdots<i_{j}} W_{i_{1}} \otimes \cdots \otimes W_{i_{j}}
$$

In the following we will use the notation $W_{I}=W_{i_{1}} \otimes \cdots \otimes W_{i_{j}}$ for a multi-index $I=\left(i_{1}, \ldots, i_{j}\right)$, with $|I|=j$, and we will denote the local coefficient system corresponding to the representation $W_{I}$ by $\mathcal{W}_{I}$.

The group $H^{*}\left(\Sigma ; \mathcal{F}_{0}\right)$ is just the ordinary real cohomology of $\Sigma$. To calculate $H^{*}\left(\Sigma^{m} ; \mathcal{F}_{j}\right)$ for $j \geq 1$ we use the fact that

$$
H^{0}\left(\Sigma ; \mathcal{W}_{I}\right)=\left\{w \in W_{I}: \xi w=w \text { for all } \xi \in \pi_{1} \Sigma\right\}
$$

the set of points in $W_{I}$ fixed by $\pi_{1} \Sigma$ (see e.g. [15, pp. 275-276]). From this we see that $H^{0}\left(\Sigma ; \mathcal{W}_{I}\right)=0$ and hence, by Poincaré duality, that $H^{2}\left(\Sigma ; \mathcal{W}_{I}\right)=0$. By induction on $m$ it follows that the cohomology of $\Sigma^{m}$ with local coefficients $\mathcal{W}_{I}$ is concentrated in dimension $m$. The same is certainly true of the cohomology of $S^{m_{1}} \Sigma \times S^{m_{2}} \Sigma$ (because the cohomology of this space is just the $S_{m_{1}} \times S_{m_{2}}$-invariant part of the cohomology of $\left.\tilde{\Sigma}^{m}\right)$, and therefore

$$
\operatorname{dim}\left(H^{m}\left(S^{m_{1}} \Sigma \times S^{m_{2}} \Sigma\right) ; \mathcal{W}_{I}\right)=\left|\chi\left(S^{m_{1}} \Sigma\right)\right|\left|\chi\left(S^{m_{2}} \Sigma\right)\right| \operatorname{dim}\left(W_{I}\right) .
$$

The Euler characteristic of $S^{m_{i}} \Sigma$ can be calculated to be $\chi\left(S^{m_{i}} \Sigma\right)=$ $(-1)^{m_{i}}\left(\begin{array}{c}2 g-2 \\ m_{i}\end{array}\right)$ (for example by Macdonald's formula [12, p.322] for the Poincaré polynomial of the symmetric product of a Riemann surface). This finishes the proof. 


\section{Calculation of the indices of the CRitical SUBMANIFOLDS}

Denote the diffeomorphism of $\mathcal{M}$ corresponding to $e^{i \theta} \in S^{1}$ by $F(\theta)$. At a fixed point $x$ we get an infinitesimal action

$$
\rho=\left.\frac{d}{d \theta} d_{x} F(\theta)\right|_{\theta=0}: T_{x} \mathcal{M} \rightarrow T_{x} \mathcal{M}
$$

As shown by Frankel [8, p.4], the fact that our Morse function is a moment map for the $S^{1}$-action, means that the indices at a critical point $x \in \mathcal{M}$ for $\mu$ can be calculated as the weights of the infinitesimal circle action $\rho$ on $T_{x} \mathcal{M}$. At a critical point $x \in \mathcal{M}$ represented by a pair $(A, \Phi)$, there are gauge transformations $g(\theta)$ such that

$$
\left(A, e^{i \theta} \Phi\right)=g(\theta) \cdot(A, \Phi)
$$

It follows from this that the weights of the action of $S^{1}$ on the tangent space $T_{x} \mathcal{M}$ are the weights of the infinitesimal gauge transformation $\psi=\left.\frac{d}{d \theta} g(\theta)\right|_{\theta=0}$. As noted in [10, p.95] there is a subspace $Y \subset T_{x} \mathcal{M}$ given by

$$
Y=H^{0}\left(\Sigma ; \operatorname{End}_{0}(E) \otimes K\right) /\left[H^{0}\left(\Sigma ; \operatorname{End}_{0}(E)\right), \Phi\right] .
$$

Because we know the weights of the action of $\psi$ on $\operatorname{End}_{0}(E)$ we can calculate the weights on $Y \subset T_{x} \mathcal{M}$ from this. To get the weights on $T_{x} \mathcal{M}$ we use the complex symplectic form $\omega$ on $\mathcal{M}$, defined by

$$
\omega\left(\left(\psi_{1}, \Phi_{1}\right),\left(\psi_{2}, \Phi_{2}\right)\right)=\int_{\Sigma} \operatorname{tr}\left(\Phi_{2} \psi_{1}-\Phi_{1} \psi_{2}\right),
$$

(cf. [10, p.90]). $Y$ is a Lagrangian subspace for $\omega$, and therefore there is an isomorphism

$$
f: T_{x} \mathcal{M} / Y \stackrel{\cong}{\longrightarrow} Y^{*}
$$

To take advantage of this we must examine the interplay between the circle action and $\omega$.

Proposition 4.1. Under the isomorphism $f$ above, a weight $m$ on $Y$ corresponds to a weight $1-m$ on $Y^{*}$.

Proof. From the definition of $\omega$ we see that $F(\theta)^{*} \omega=e^{i \theta} \omega$ at a critical point. For $v, w \in T_{m} \mathcal{M}$ differentiating this identity gives

$$
\begin{aligned}
i \omega(v, w) & =\left.\frac{d}{d \theta} \omega(d g(\theta) v, d g(\theta) w)\right|_{\theta=0} \\
& =\omega(\rho(v), w)+\omega(v, \rho(w)) .
\end{aligned}
$$

The proposition follows easily from this.

Proposition 4.2. The critical submanifolds for the function $\mu$ have the following indices.

(i) For $\frac{1}{3} d<l<\frac{1}{3} d+g-1$ the index of $N\left(l-\frac{1}{3} d\right)$ is $\lambda_{l}=2(3 l-$ $d+2 g-2)$. 
(ii) For $\frac{1}{3} d+1-g<\bar{l}<\frac{1}{3} d$ the index of $N\left(\frac{1}{3} d-\bar{l}\right)$ is $\lambda_{\bar{l}}=2(d-$ $3 \bar{l}+2 g-2)$.

(iii) For $0 \leq m<4(g-1)$ the index of $N(4(g-1)-m)$ is $\lambda_{m}=$ $8(g-1)-2 m$

Proof. We shall only prove (i), the other cases being very similar. Let $(E, \Phi)$ represent a point of $N\left(l-\frac{1}{3} d\right)$. Then it is of the form $E=L \oplus V$, with $\operatorname{rk}(L)=1, \operatorname{rk}(V)=2$, and $\Phi=\left(\begin{array}{ll}0 & 0 \\ \phi & 0\end{array}\right)$. The bundle $\operatorname{End}_{0}(E)$ splits in the following way:

$$
\operatorname{End}_{0}(E)=\operatorname{Hom}(L, V) \oplus \operatorname{Hom}(V, L) \oplus \operatorname{End}(V) .
$$

Because $\operatorname{tr}(\psi)=0$ we see that $\psi$ acts with weights $-\frac{2}{3}$ on $L$ and $\frac{1}{3}$ on $V$. It follows that $\psi$ acts with weights $(1,-1,0)$ with respect to the decomposition above. From Proposition 4.1 the weights on $Y^{*}$ are $(0,2,1)$, and in particular we see that there are no negative weights on $Y^{*}$. We also have to examine $\left[H^{0}\left(\Sigma ; \operatorname{End}_{0}(E)\right), \Phi\right]$. This consists of elements of the form

$$
\left[\left(\begin{array}{cc}
-\operatorname{tr}(a) & b \\
c & a
\end{array}\right),\left(\begin{array}{ll}
0 & 0 \\
\phi & 0
\end{array}\right)\right]=\left(\begin{array}{cc}
b \phi & 0 \\
a \phi+\phi \operatorname{tr}(a) & -\phi b
\end{array}\right),
$$

where $b \in H^{0}\left(\Sigma ; V^{*} \otimes L\right)$ and $a \in H^{0}(\Sigma ; \operatorname{End}(V))$. Clearly $b_{1} \phi=b_{2} \phi$ if and only if $b_{1}=b_{2}$, so

$$
H^{0}\left(\Sigma ; V^{*} \otimes L\right) \hookrightarrow\left[H^{0}\left(\Sigma ; \operatorname{End}_{0}(E)\right), \Phi\right]
$$

is injective. Any $a \in H^{0}(\Sigma ; \operatorname{End}(V))$ such that $a \phi+\operatorname{tr}(a) \phi=0$ has $\tilde{L}$ as an eigenspace with eigenvalue $-\operatorname{tr}(a)$, which is constant, being a globally defined holomorphic function. It follows that the other eigenvalue of $A$ is also constant, and equal to $2 \operatorname{tr}(a)$. So if $\operatorname{tr}(a) \neq 0$, we get a decomposition $V=\tilde{L} \oplus L^{\perp}$. This is impossible, so $\operatorname{tr}(a)=0$ and hence $a=0$. It follows that

$$
H^{0}(\Sigma ; \operatorname{End}(V)) \hookrightarrow\left[H^{0}\left(\Sigma ; \operatorname{End}_{0}(E)\right), \Phi\right]
$$

is injective. Thus

$$
\left[H^{0}\left(\Sigma ; \operatorname{End}_{0}(E)\right), \Phi\right] \cong H^{0}(\Sigma ; \operatorname{End}(V)) \oplus H^{0}(\Sigma ; \operatorname{Hom}(V, L)),
$$

where the first summand lies in $H^{0}(\Sigma ; \operatorname{Hom}(L, V) \otimes K)$, and the second lies in $H^{0}(\Sigma ; \operatorname{End}(V) \otimes K)$.

Thus the index is equal to $\operatorname{dim}_{\mathbb{R}} H^{0}(\Sigma ; \operatorname{Hom}(V, L) \otimes K)$. Now note that the corresponding $H^{1}$ vanishes: it is Serre dual to $H^{0}(\Sigma ; \operatorname{Hom}(L, V))$, so suppose we had a non-zero element of this. This would define a non-trivial map $L \rightarrow L^{\prime \prime} \subset V$ for some line bundle $L^{\prime \prime}$, and hence $-\operatorname{deg}(L)+\operatorname{deg}\left(L^{\prime \prime}\right) \geq 0$. On the other hand $\operatorname{deg}\left(L^{\prime \prime}\right)<\frac{1}{3} d$ by stability, and we also know that $\operatorname{deg}(L)>\frac{1}{3} d$. Hence $-\operatorname{deg}(L)+\operatorname{deg}\left(L^{\prime \prime}\right)<0$, which is a contradiction. From Riemann-Roch we then get

$$
\begin{aligned}
\lambda_{l} & =2(-v+2 l+4(g-1)+2(1-g)) \\
& =2(3 l-d+2(g-1)),
\end{aligned}
$$


which finishes the proof.

\section{REFERENCES}

1. M. F. Atiyah and R. Bott, The Yang-Mills equations over Riemann surfaces, Philos. Trans. Roy. Soc. London Ser. A 308 (1982), 523-615.

2. S. B. Bradlow, Special metrics and stability for holomorphic bundles with global sections, J. Differential Geom. 33 (1991), 169-213.

3. S. B. Bradlow and G. D. Daskalopoulos, Moduli of stable pairs for holomorphic bundles over Riemann surfaces, Internat. J. Math. 2 (1991), 477-513.

4. S. B. Bradlow, G. D. Daskalopoulos, and R. A. Wentworth, Birational equivalences of vortex moduli, Preprint, 1993.

5. K. Corlette, Flat g-bundles with canonical metrics, J. Diff. Geom. 28 (1988).

6. U. V. Desale and S. Ramanan, Poincaré polynomials of the variety of stable bundles, Math. Ann. 216 (1975), 233-244.

7. S. K. Donaldson, Twisted harmonic maps and the self-duality equations, Proc. London Math. Soc. (3) 55 (1987), 127-131.

8. T. Frankel, Fixed points and torsion on Kähler manifolds, Ann. of Math. (2) 70 (1959), 1-8.

9. G. Harder and M. S. Narasimhan, On the cohomology groups of moduli spaces of vector bundles on curves, Math. Ann. 212 (1975), 215-248.

10. N. J. Hitchin, The self-duality equations on a Riemann surface, Proc. London Math. Soc. (3) $\mathbf{5 5}$ (1987), 59-126.

11. __ Lie groups and Teichmüller space, Topology 31 (1992), 449-473.

12. I. G. Macdonald, Symmetric products of an algebraic curve, Topology 1 (1962), 319-343.

13. C. T. Simpson, Constructing variations of Hodge structure using Yang-Mills theory and applications to uniformization, J. Amer. Math. Soc. 1 (1988), 867918.

14. M. Thaddeus, Stable pairs, linear systems and the Verlinde formula, to appear in Inv. Math.

15. G. W. Whitehead, Elements of homotopy theory, Springer-Verlag, 1978.

Mathematics Institute, University of Warwick, Coventry CV4 7AL, U.K.

E-mail address: pbg@maths.warwick.ac.uk 